Textures and Microstructures, 1989, Vol. 10, pp. 361-373

Reprints available directly from the publisher

Photocopying permitted by license only

(C) 1989 Gordon and Breach Science Publishers Inc.

Printed in the United Kingdom

\title{
Fast Texture Measurements Using a Position Sensitive Detector
}

\author{
DORTE JUUL JENSEN and TORBEN LEFFERS \\ Metallurgy Department, Risø National Laboratory, Roskilde, Denmark
}

(Received 15 February, 1989)

A technique for fast texture determination by neutron diffraction is described. With the technique a complete texture analysis requires from 15 to 45 minutes measuring time and the kinetics of the development in single texture components can be studied with a time resolution of the order of seconds. It is shown how these two measuring principles can be used for in-situ kinetic investigations of recrystallization. Independent of speed, texture measurement by neutron diffraction has an advantage in improved statistics which is examplified by a series of measurements on the early stage of texture development in copper and brass.

KEY WORDS: Neutron diffraction, pole figure measurement, position sensitive detector, recrystallization, kinetics, in-situ investigation

\section{INTRODUCTION}

Standard techniques for the registration of pole figures are too time-consuming to be applied for on-line kinetic studies of fast processes, and more rapid data acquisition is required. In 1981 the potential of neutron diffraction as a tool for investigating recrystallization kinetics of individual texture components was demonstrated by Hansen et al. (1981). One of the later developments in the field is the use of multidetectors, and Juul Jensen and Kjems (1983) have shown that the data collection rate can be greatly enhanced by the use of a linear position-sensitive detector.

In section 2 of this paper an experimental set-up for fast and automatic texture determination by neutron diffraction is described. In Section 3, examples of applications are given. 


\section{EXPERIMENTAL TECHNIQUE}

\subsection{The spectrometer}

The basic unit for the fast neutron texture measurements is the double axis spectrometer TAS 3, placed at the Ris $\varnothing$ DR3 reactor. The spectrometer is equipped with a linear position sensitive detector, and an Euler goniometer (Figure 1). A given wavelength is selected from the incident continuous neutron spectrum by a curved pyrolytic graphite crystal monochromater producing a vertically focussed monochromatic beam on the sample (Figure 2). The sample is mounted in the Euler goniometer, where it can be placed in any position relative to the incident and diffracted beam by computer control. The intensity of the diffracted beam is recorded

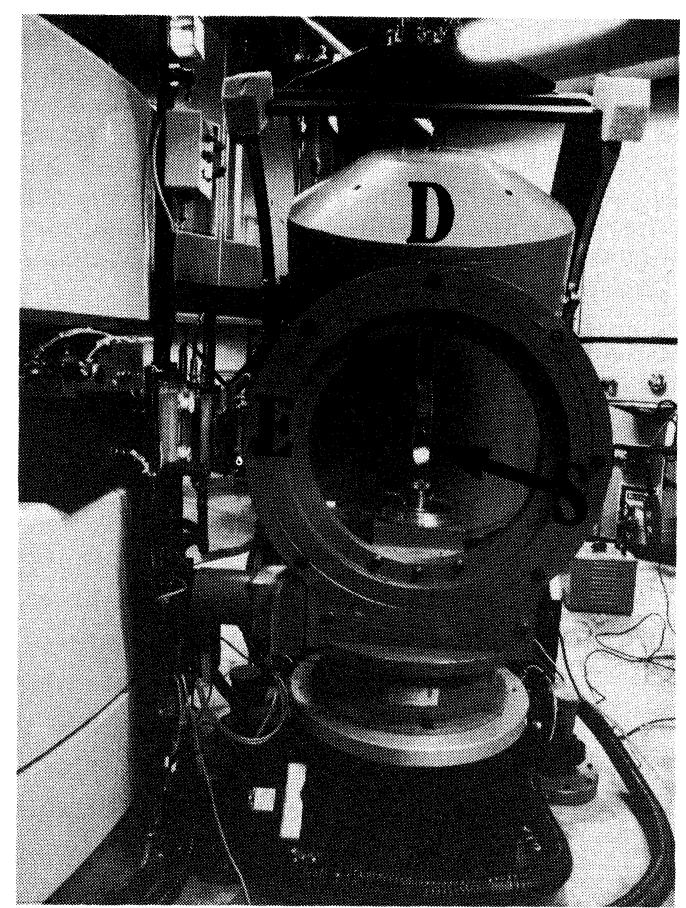

Figure 1 The neutron texture spectrometer. The Euler goniometer, the sample and the detector (inside a heavy boron-plastic shielding) are marked by E, S and D, respectively. 


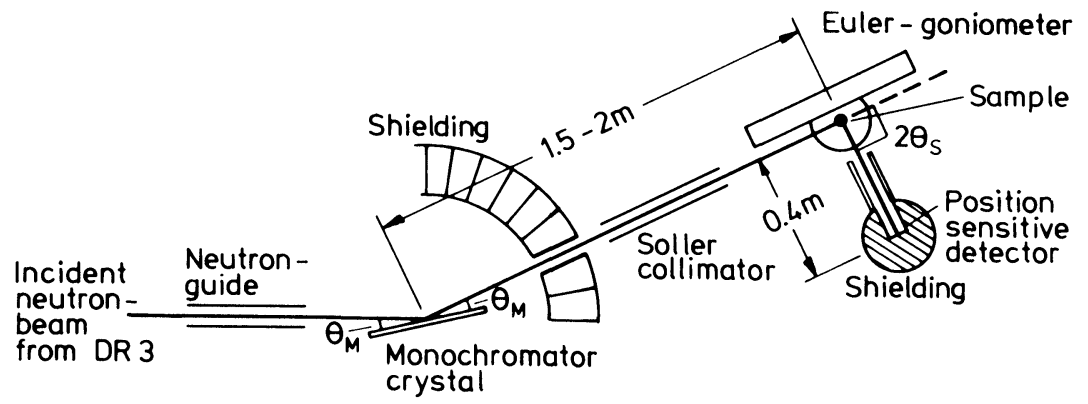

Figure 2 The experimental set-up (horizontal section).

in a $50 \mathrm{~cm}$ long linear position sensitive detector placed with its axis vertical to detect the intensity simultaneously along $\sim 50^{\circ}$ of a Debye-Scherrer ring. A given diffraction condition-i.e. a given angle between the incident and the diffracted beam-is only fulfilled for the entire detector when $2 \theta$ is equal to $90^{\circ}$ (Figure 3). The continuous spectrum from a thermal reactor, however, allows a selection of a wavelength which makes $2 \theta=90^{\circ}$ for most low-index reflections from common metals and alloys.

With this detector system a considerable enhancement of the data collection rate is obtained compared to a set-up with a "point-

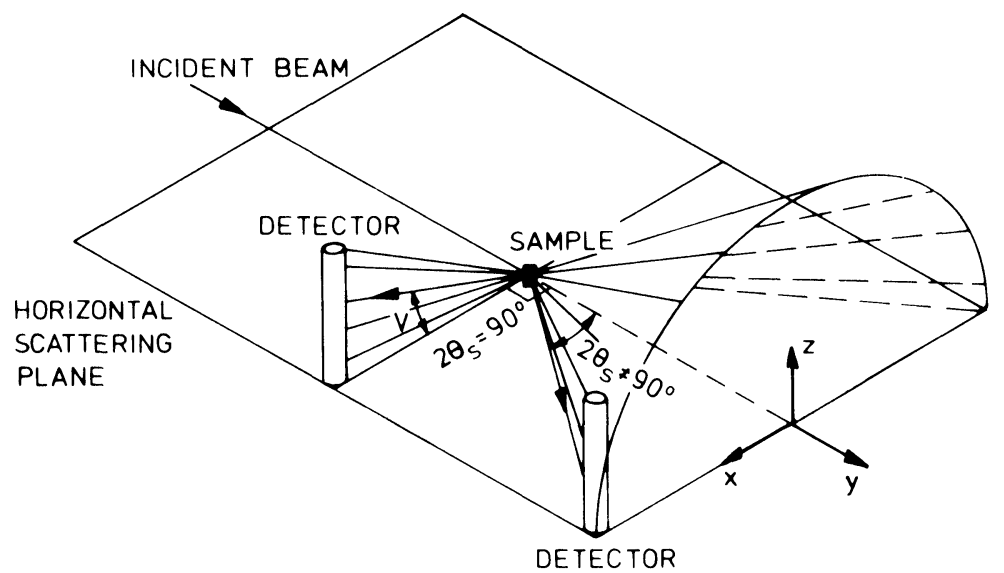

Figure 3 Illustration of the use of a linear position sensitive detector to measure the intensity distribution along a (hkl) Debye-Scherrer cone; at $2 \theta_{s}=90^{\circ}$ the cone degenerates to a plane, and the detector axis lies completely in this plane. 
detector". The data recorded in the detector are compressed into 18 "reduced channels" representing equivalent segments along the detector. This means that the position sensitive detector system can be regarded as 18 independent detectors placed above each other along the Debye-Scherrer ring. (For further experimental details, see Juul Jensen and Kjems, 1983).

The samples for the neutron texture measurements are normally cylindrical with a total volumen between 0.1 and $2 \mathrm{~cm}^{3}$. For heavily rolled materials this relative large sample volumen is achieved by stacking a number of circular discs on top of each other. As the penetration depth of neutrons is large in most of the common metals and alloys (several centimeters), the measured textures are true averages of the sample textures.

A furnace is available for annealing samples in the spectrometer (see Figure 4). In the furnace the samples are normally kept in vacuum and temperatures up to $2000^{\circ} \mathrm{C}$ can be reached. A typical time for heating up to $500^{\circ} \mathrm{C}$ is five minutes after which the

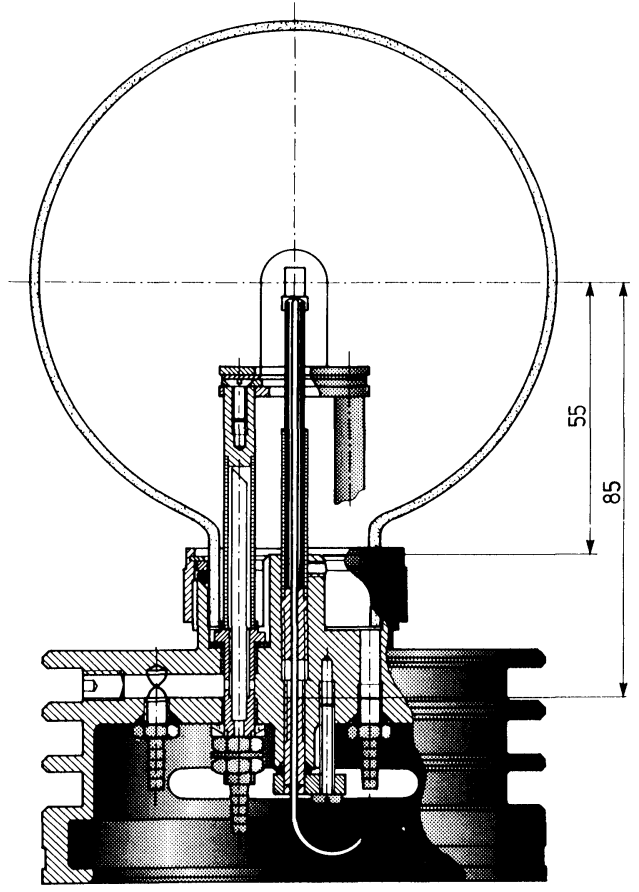

Figure 4 The texture-furnace. 
temperature can be kept constant to within a few degrees. The temperature is measured with a thermocouple, normally chromelalumel, placed in the centre of the sample.

The complete spectrometer, the temperature and the processing of the signals from the position sensitive detector system are under full automatic control by a PDP-11/23 computer. The measured data are stored on hard or floppy discs, and they can be analyzed at any PDP-11 computer or transferred to a more powerful computer. A Canon computer with a colour screen and an 8-colour jet-ink plotter is directly coupled to the PDP-11 providing an on-line graphical presentation of the measured data.

\subsection{Experimental procedure}

With one setting of the sample the position sensitive detector records 18 values of diffracted intensities (Figure 5a). This corresponds to measurements along $\sim 34^{\circ}$ of a small circle in a pole figure (Figure $5 \mathrm{~b}$ ). A complete pole figure, or any part of it, is obtained by rotating the sample discontinuously in the Euler goniometer, with the sample fixed while counting. Typically, 180

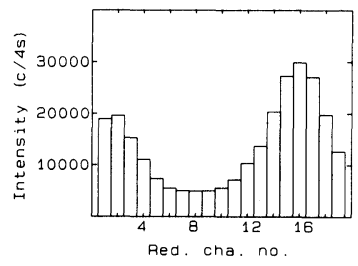

a

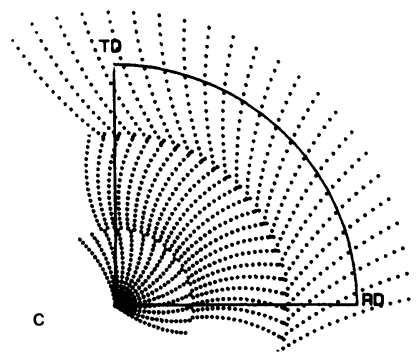

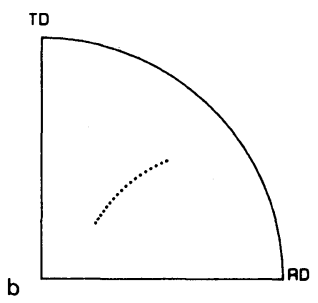

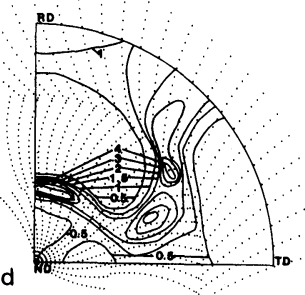

Figure 5 (a) Example of measured intensities along the detector. (b) With one sample setting the detector covers $34^{\circ}$ of a small circle in the pole figure plane. (c) The sampling mesh. (d) Example of a measured pole figure. Contour lines are used to identify the different pole density levels. 
settings of the sample, corresponding to $180 \times 18$ measuring points, are required for a complete pole figure measurement. However, owing to symmetry this number can, in general, be reduced. For example, for symmetrically rolled materials only a quarter pole figure is needed. In this case 61 settings of the sample is used to obtain the sampling mesh shown in Figure 5c. An example of a measured quarter pole figure is shown in Figure 5d. Typical measuring times for a quarter pole figure (with an accuracy of 2-3\% as determined by counting statistics) are in the range 7 to 15 minutes depending on the scattering cross section of the sample material.

A typical time resolution for determination of two pole figures which can be used for ODF-calculations is therefore about 20 minutes. Thus, it is possible to make reasonably accurate ODFbased kinetic investigations of processes, which involve a texture transformation over a couple of hours.

The kinetics of very fast processes, where this time resolution is insufficient, can be followed by means of partial pole figures that focus on selected texture components. Here the sample orientation is changed only between a few preselected positions. In the limiting case the sample is kept fixed during the measurements: since the position sensitive detector covers $34^{\circ}$ of a small circle in the pole figure plane (Figure 5b), it is possible to position the sample so that the detector records the change in intensity of one or even a few texture components simultaneously, and thus a time resolution of the order of seconds is obtained.

\section{APPLICATIONS}

\subsection{Recrystallization of aluminium}

During recrystallization nuclei develop and grow at the expense of the deformed matrix. In general both these basic processes are orientation dependent, resulting in a change in texture from the deformed to the recrystallized state. An example is given in Figure 6. The ODF for commercially pure aluminium cold rolled to $95 \%$ reduction in thickness is shown in Figure 6a. The ODF for the same material after complete recrystallization is shown in Figure 6b. It is 

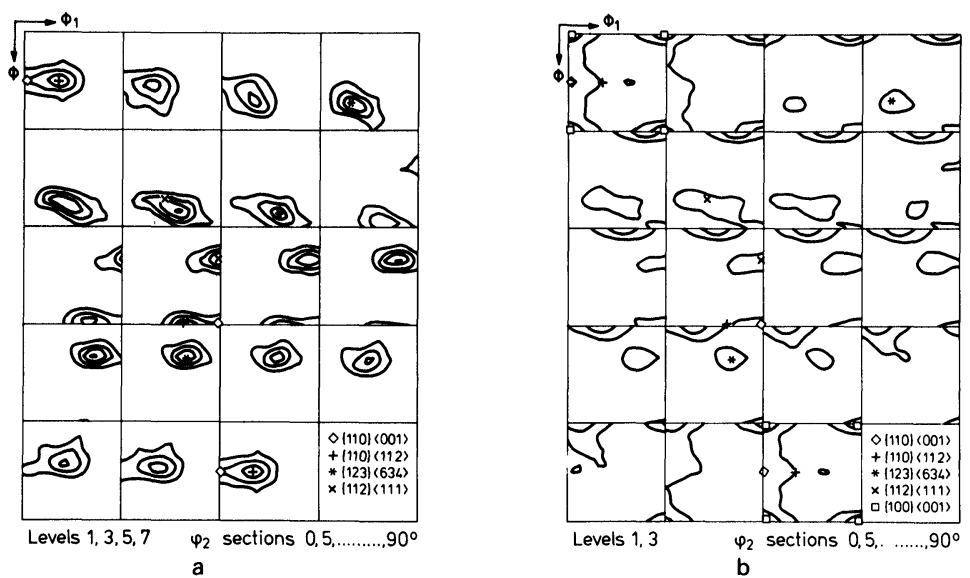

Figure 6 ODFs for $99.6 \% \mathrm{Al}$. (a) Cold rolled to $95 \%$ reduction in thickness. (b) Cold rolled to $95 \%$ reduction and annealed at $240^{\circ} \mathrm{C}$ for 15 hours.

seen that the deformation texture consists of an orientation tube running through the $\varphi_{2}$-sections of the Euler space close to the $\{110\}\langle 112\rangle,\{123\}\langle 634\rangle$ and $\{112\}\langle 111\rangle$ plus some $\{110\}\langle 001\rangle$ texture.

In the recrystallized state the texture consists of cube component, retained rolling texture components and a relative large volume fraction of other texture components distributed apparently randomly over the Euler space (Figure 6b).

This texture transformation from the deformed to the recrystallized state is rather complex and a complete ODF investigation is needed for determination of the overall kinetics. From ODF data measured on-line during annealing, the volume fraction of the five main texture components is calculated by integration over 15 degrees around each component (Figure 7). This type of data can be used to obtain information about how the recrystallization texture components strengthen at the expense of the different rolling components. Combined with microscopial observations the kinetic texture data have given an improved understanding of both the nucleation and growth processes taking place in the deformed material (Juul Jensen, Hansen and Humphreys, 1985; Hansen and Juul Jensen, 1986). Furthermore, the results can be used to evaluate kinetic models. As an illustration of this the measured data (Figure 


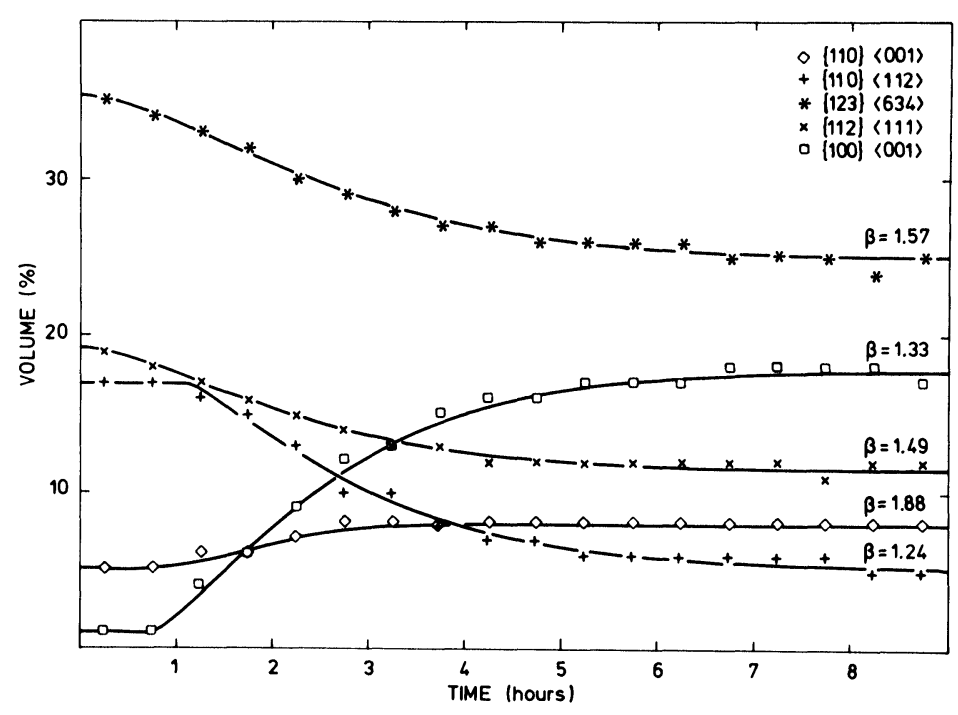

Figure 7 The development of specific texture components measured in-situ during isothermal annealing at $240^{\circ} \mathrm{C}$ of $99.6 \% \mathrm{Al}$ cold rolled to $95 \%$ reduction in thickness.

7) are fitted to an Avrami type of equation (Avrami, 1940):

$$
X=X_{\infty}-\left(X_{\infty}-X_{0}\right) \exp \left(-A\left(t-t_{0}\right)^{\beta}\right) \text { for } t>t_{0}
$$

where $X, X_{\infty}, X_{0}$ are the volume fraction of a given texture component at time $t$, infinity and zero (start of annealing), respectively, $t_{0}$ is an incubation time, and $A, \beta$ are constants characteristic of the process. It can be seen in Figure 7 that the equation gives a reasonable fit to the measured points and thereby an accurate determination of the fitted parameters, $A, \beta$.

For investigations of fast processes or for obtaining more precise information about specific events happening during a slower process, e.g. for investigations of incubation periods, the partial pole figure technique is used. For example the development in the $\{100\}\langle 001\rangle$ and the $\{123\}\langle 634\rangle$ components have been followed by measuring a partial (200) pole figure keeping the sample fixed so that the detector covered the two components during annealing. A typical result is shown in Figure 8. Here the peak at channel 0 corresponds to $\{100\}\langle 001\rangle$ and the peak at channel 15 to $\{123\}\langle 634\rangle$. At time 


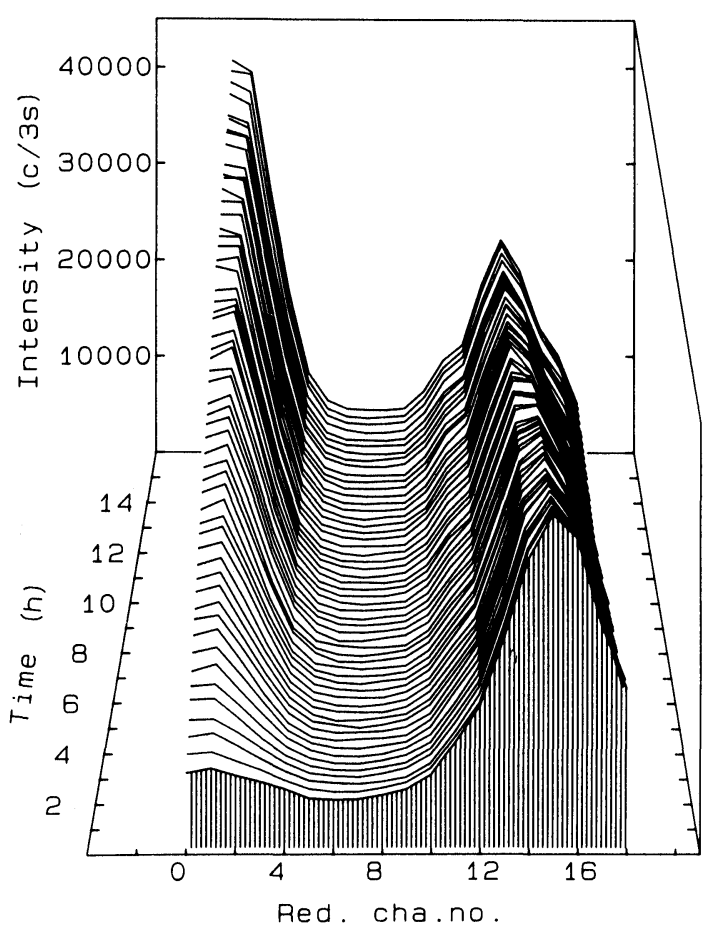

Figure 8 The development in time of intensities measured along the detector with the sample fixed in one position during annealing of pure copper.

zero a strong $\{123\}\langle 634\rangle$ peak is seen, and as the annealing time increases this peak decreases, while the $\{100\}\langle 001\rangle$ peak increases. By spatial integration over each of these peaks the development in the two components can be followed (Figure 9). Based on this type of data the incubation periods can be determined very accurately.

In the derivation of Eq. (1) rather simple assumptions about the nucleation and growth process were used. To be able to consider more complex processes and to get more detailed information about the recrystallization, for example the microstructural development, computer simulations can be used. As it can be seen in Figure 7 and 9 the nucleation and growth characteristics of the different texture components in commercially pure aluminium is not identical. A 


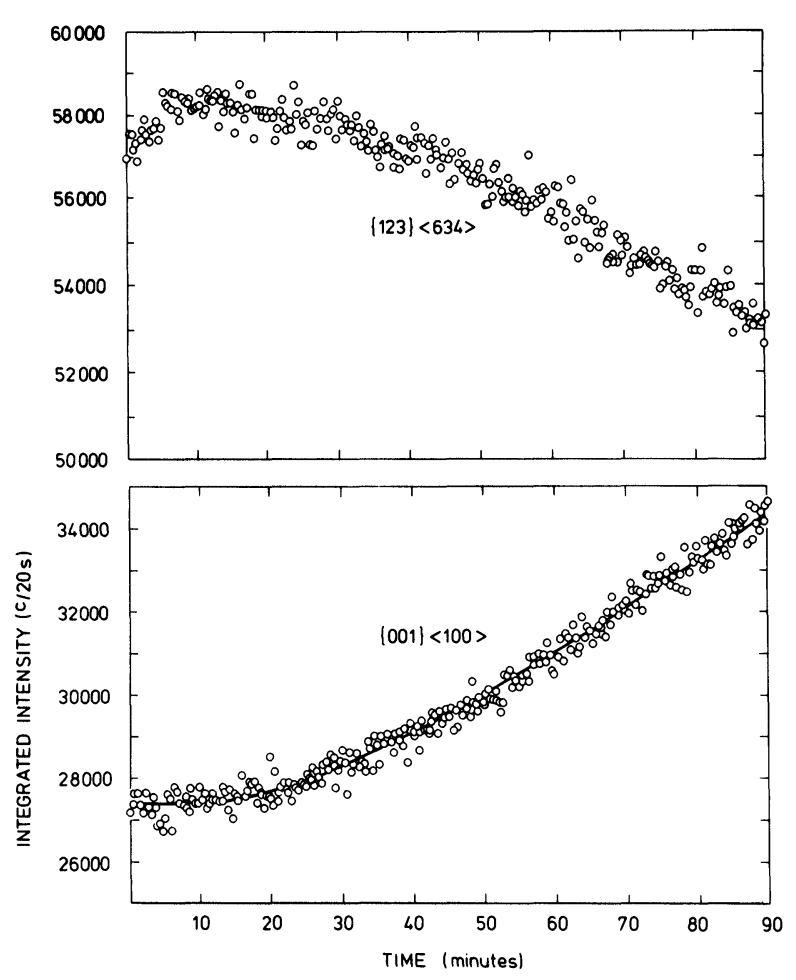

Figure 9 Kinetic curves for the initial evolution of the $\{100\}\langle 001\rangle$ and $\{123\}\langle 634\rangle$ texture component in $99.6 \% \mathrm{Al}$ cold rolled $90 \%$ measured during annealing at $253^{\circ} \mathrm{C}$. The full line in the bottom figure is a best fit of the experimental points to Eq. (1).

computer model which operate with three types of grains (i.e. three recrystallized texture components) has been developed (Juul Jensen and Hansen, 1986). Reasonable agreement between the model calculations and the experimental results for the volume fraction recrystallized as a function of time, the average grain size and the grain size distribution was obtained.

\subsection{Recrystallization of copper}

When fitted with an Avrami equation (Eq. (1)), the kinetics of the recrystallization of heavily rolled copper are characterized by very 


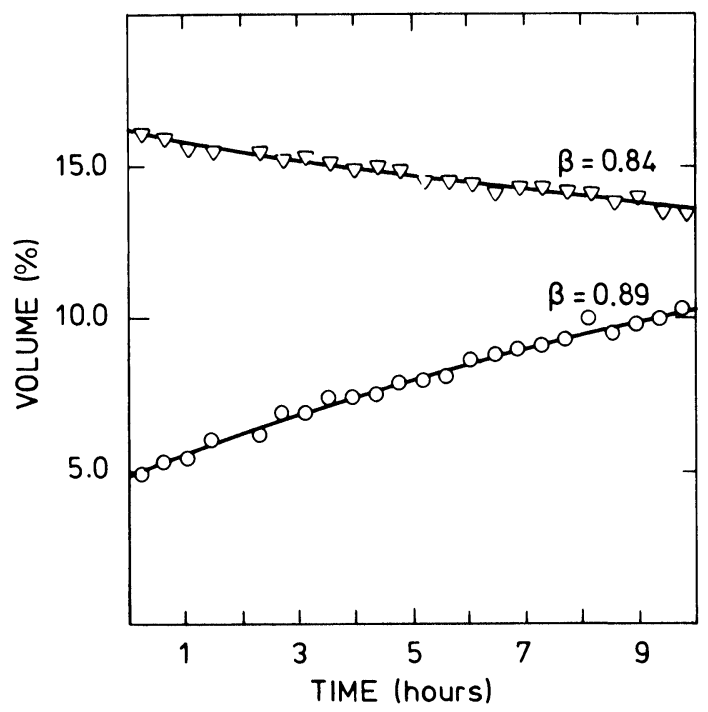

Figure 10 Initial kinetics of recrystallization in heavily cold-rolled pure copper annealed at $135^{\circ} \mathrm{C}$ as represented by the change in volume fraction of the components $\{112\}\langle 111\rangle(\nabla)$ and $\{100\}\langle 001\rangle(O)$.

low $\beta$ values - of the order of 1 (Figure 10) (Hansen, Leffers, Kjems, 1981, Juul Jensen, Hansen, Kjems, Leffers, 1984). The standard interpretation of $\beta \sim 1$ in the Avrami equation is onedimensional growth. However, this obviously does not apply to the recrystallizing grains: they are approximately equiaxed. Since the kinetic measurements are quite precise an interpretation outside the range of interpretations considered by Avrami must be sought.

By computer modeling it was shown (Leffers, 1986) that the only simple model based on isotropic behaviour capable of explaning the low $\beta$ values was a model with approximately constant rate of nucleation of grains which then very rapidly grew to their final size. The microstructural interpretation of this model is that the nuclei form in clusters and hence rapidly form islands of recrystallized material which subsequently grow rather slowly.

\subsection{Early stage of development of rolling texture}

Neutron diffraction has the general advantage over X-ray diffraction for texture analysis that it is a bulk measurement, i.e. that the 


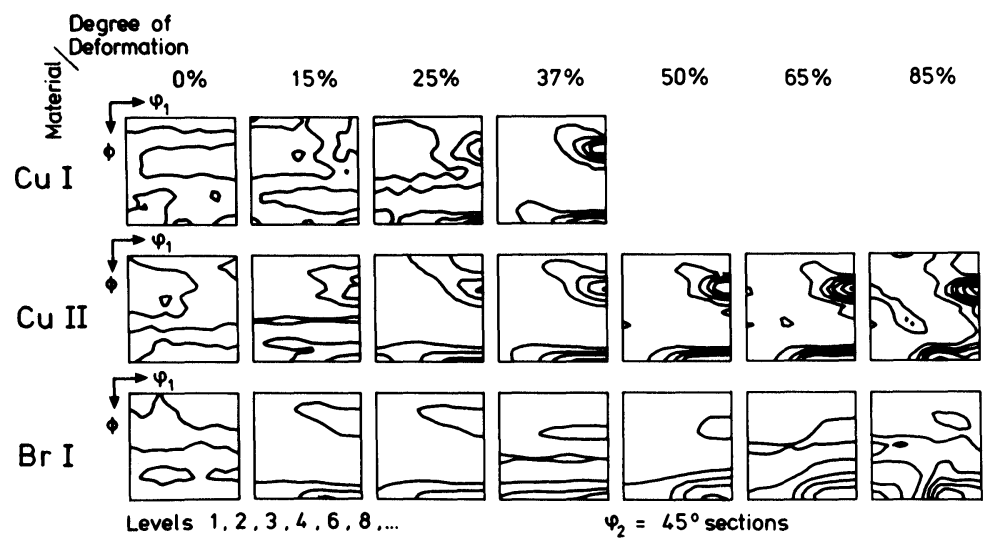

Figure 11 ODF development with increasing degree of deformation for two batches of copper and one batch of brass $(15 \% \mathrm{Zn})$. CuII and BrI have only weak initial textures while $\mathrm{CuI}$ has an initial texture which deviates significantly from random. Particularly for CuII and BrI there is a clear difference in texture development from the very early stage.

number of grains monitored is greater. This is known to be important for coarse-grained materials. However, the advantage of the improved statistics is also significant for investigations of the early stage of development of deformation texture in normal fine-grained materials.

The traditional interpretation of the early stages of the development of the copper-type and the brass-type rolling texture, based on $\mathrm{X}$-ray diffraction, is that the difference does not appear before reductions of about $50 \%$ are reached. With neutron diffraction there is less scatter in the textures registered for low degrees of reduction, and it is evident that there is a difference in texture development between copper and brass from the very beginning as illustrated in Figure 11.

\section{CONCLUDING REMARKS}

A fast and precise in-situ determination of the texture development in bulk specimens is possible with the described neutron diffraction technique. Combined with microstructural investigations the results 
have given an improved understanding of many of the micromechanisms that operate in various materials, e.g. during recrystallization. Of course the technique also incorporates the standard advantage of neutron diffraction, viz. the improved statistics associated with the great penetration of neutrons.

\section{References}

Avrami, M. (1940). J. Chem. Phys. 8, 212.

Hansen, N., Leffers, T. and Kjems, J. K. (1981). Acta Met. 29, 1523-1533.

Hansen, N. and Juul, Jensen, D. (1986). Met. Trans 17A, 253-259.

Juul Jensen, D. and Hansen, N. (1986). In: Annealing Processes-Recovery, Recrystallization and Grain Growth. (Eds: N. Hansen et al.) RIS $\varnothing$ National Laboratory, Roskilde, 379-384.

Juul, Jensen, D., Hansen, N. and Humphreys, F. J. (1985). Acta Met. 33, 2155-2162.

Juul Jensen, D., Hansen, N., Kjems, J. K. and Leffers, T. (1984). In: Proc. of ICOTOM 7. Eds: P. Jongenburger et al., Netherlands Society for Materials Science, Zwijndrecht, 777-782.

Juul Jensen, D. and Kjems, J. K. (1983). Textures and Microstructures 5, 239-245.

Leffers, T. (1986). In: Annealing Processes-Recovery, Recrystallization and Grain Growth. (Eds: N. Hansen et al.). RIS $\varnothing$ National Laboratory, Roskilde, 427-436.

Leffers, T. and Juul Jensen, D. (1986). Textures and Microstructures 8+9, 467-480. 